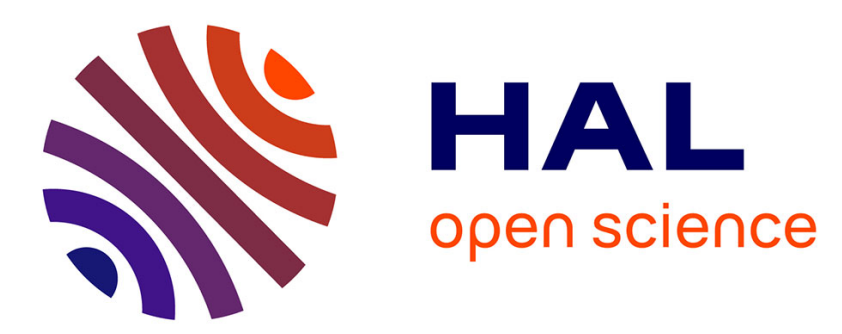

\title{
Fracture of disordered solids in compression as a critical phenomenon: III. Analysis of the localization transition
}

\author{
Renaud Toussaint, Steven R Pride
}

\section{To cite this version:}

Renaud Toussaint, Steven R Pride. Fracture of disordered solids in compression as a critical phenomenon: III. Analysis of the localization transition. Physical Review E : Statistical, Nonlinear, and Soft Matter Physics, 2002, 66 (3), pp.036137. 10.1103/physreve.66.036137 . hal-00110574

\section{HAL Id: hal-00110574 https://hal.science/hal-00110574}

Submitted on 20 Nov 2018

HAL is a multi-disciplinary open access archive for the deposit and dissemination of scientific research documents, whether they are published or not. The documents may come from teaching and research institutions in France or abroad, or from public or private research centers.
L'archive ouverte pluridisciplinaire HAL, est destinée au dépôt et à la diffusion de documents scientifiques de niveau recherche, publiés ou non, émanant des établissements d'enseignement et de recherche français ou étrangers, des laboratoires publics ou privés. 


\title{
Fracture of disordered solids in compression as a critical phenomenon. III. Analysis of the localization transition
}

\author{
Renaud Toussaint* and Steven R. Pride ${ }^{\dagger}$ \\ Géosciences Rennes, Université de Rennes 1, 35042 Rennes Cedex, France
}

(Received 14 November 2001; revised manuscript received 13 June 2002; published 27 September 2002)

\begin{abstract}
The properties of the Hamiltonian developed in Paper II are studied showing that at a particular strain level a "localization" phase transition occurs characterized by the emergence of conjugate bands of coherently oriented cracks. The functional integration that yields the partition function is then performed analytically using an approximation that employs only a subset of states in the functional neighborhood surrounding the most probable states. Such integration establishes the free energy of the system, and upon taking the derivatives of the free energy, the localization transition is shown to be continuous and to be distinct from peak stress. When the bulk modulus of the grain material is large, localization always occurs in the softening regime following peak stress, while for sufficiently small bulk moduli and at sufficiently low confining pressure, the localization occurs in the hardening regime prior to peak stress. In the approach to localization, the stress-strain relation for the whole rock remains analytic, as is observed both in experimental data and in simpler models. The correlation function of the crack fields is also obtained. It has a correlation length characterizing the aspect ratio of the crack clusters that diverges as $\xi \sim\left(\boldsymbol{\varepsilon}_{c}-\boldsymbol{\varepsilon}\right)^{-2}$ at localization.
\end{abstract}

DOI: 10.1103/PhysRevE.66.036137 PACS number(s): 62.20.Mk, 46.50. $+\mathrm{a}$, 46.65. $+\mathrm{g}, 64.60 . \mathrm{Fr}$

\section{INTRODUCTION}

In Paper II of this series, we obtained the Hamiltonian $E_{j}\left(\boldsymbol{\varepsilon}, \boldsymbol{\varepsilon}_{m}\right)$ of a population of interacting cracks which is the energy necessary to lead a mesovolume of a disordered-solid system from uncracked and unstrained initial conditions, to a final crack state $j$ at a maximum imposed strain $\boldsymbol{\varepsilon}_{m}$ that is possibly different than the actual strain $\boldsymbol{\varepsilon}$ if the system has been subsequently unloaded. Using this Hamiltonian, we prove here that at a well-defined strain $\boldsymbol{\varepsilon}_{c}$, the system undergoes a phase transition to bands of coherently oriented cracks.

To study the nature of this localization transition, we must evaluate the partition function $Z$ from which all physical properties depending on the crack distribution are obtained through differentiation. In Paper I, it was established that $Z$ takes a standard form

$$
Z\left(\boldsymbol{\varepsilon}, \boldsymbol{\varepsilon}_{m}, T\right)=\sum_{j} e^{-E_{j}\left(\boldsymbol{\varepsilon}, \boldsymbol{\varepsilon}_{m}\right) / T}
$$

despite the fact that it derives from the initial quenched disorder in the grain-contact strengths and has nothing to do with fluctuations through time. The possible crack states $j$ for a mesovolume are defined by a local order parameter $\varphi(\boldsymbol{x})$ distributed at each cell $\boldsymbol{x}$ of a regular square network of identical cells. The amplitude of $\varphi(\boldsymbol{x})$ corresponds to the length of a local crack (always less than cell dimensions), and its sign indicates its orientation $\left( \pm 45^{\circ}\right.$ relative to the principalstress axis).

\footnotetext{
*Present address: Department of Physics, University of Oslo, P.O. Box 1048 Blindern, 0316 Oslo 3, Norway. Email address: Renaud.Toussaint@fys.uio.no

${ }^{\dagger}$ Email address: Steve.Pride@univ-rennes1.fr
}

Our approach for performing the sum over states begins by determining which fields $\varphi$ maximize the Hamiltonian. Because the temperature in strain-controlled experiments is negative, such maximizing states are the dominant terms in Eq. (1). Any change in the nature of the maximizing crack fields or in the nature of the Hamiltonian in their neighborhood (e.g., the vanishing of a second derivative) corresponds to a phase transition.

In Sec. II, the localization transition is identified and the geometrical nature of the crack fields in the "functional neighborhood" surrounding the maximizing states defined. In Sec. III, we sum only over this subset of all states to obtain an analytical approximation of $Z$. In Sec. IV, the free energy $F=-T \ln Z$ is differentiated with respect to $\varepsilon$ and $T$ to determine both the sustained stress $\tau$, the energy $U$, and the entropy $S$. In the approach to localization, no singularities are present in either $F$ or any of its derivatives with respect to strain or temperature which demonstrates, among other things, that the stress/strain relation is analytic up to (and including) localization. In Sec. V, an external field $J$ is introduced that couples to $\varphi$ permitting an autocorrelation function to be obtained. All singularities at localization are in the second (and higher) derivatives of $F$ with respect to $J$ with the consequence that the correlation length diverges as $\xi$ $\sim\left(\boldsymbol{\varepsilon}_{c}-\boldsymbol{\varepsilon}\right)^{-2}$.

\section{PRINCIPLE OF THE TRANSITION}

\section{A. Extrema of the Hamiltonian}

We now determine the most probable states by maximizing the Hamiltonian $E_{j}\left(\boldsymbol{\varepsilon}, \boldsymbol{\varepsilon}_{m}\right)$ along the load path $\boldsymbol{\varepsilon}=\boldsymbol{\varepsilon}_{m}$. From the summary of Paper II, we have

$$
E_{j}=E^{0}\left(\boldsymbol{\varepsilon}_{m}\right)+(1-q)\left\{E^{\mathrm{av}}\left(\boldsymbol{\varepsilon}_{m}\right)[\varphi]+E^{\mathrm{int}}\left(\boldsymbol{\varepsilon}_{m}\right)[\varphi]\right\},
$$

where $E^{0}$ is the energy of the intact material, $E^{\text {av }}$ is the energy due to the crack field when crack interactions are 
neglected, and $E^{\text {int }}$ is the energy due to crack interactions. The parameter $q$ derives from the quenched-disorder distribution and is bounded as $1 / 2 \leqslant q<1$.

That the Hamiltonian must be maximized and not minimized comes from the temperature parameter being negative as was quantitatively established in Sec. IV of Paper I. Because we assume the system is intact before strain is applied, it is a fact of our model that the intact state is always the most probable. For this to hold true, the temperature must be negative in strain-controlled experiments because the arrival of cracks at constant strain always reduces the energy in a mesovolume.

\section{Mean-field terms}

A mean-field simplification of the model built in Paper II would reduce the Hamiltonian to the sole term

$$
\begin{aligned}
E^{0}+(1-q) E^{\mathrm{av}}= & \frac{1}{2}\left[\alpha \Delta^{2}+(1-\alpha) \gamma^{2}\right] \\
& -(1-q) \epsilon \bar{\psi}\left[\kappa_{2} \Delta^{2}+\kappa_{3} \gamma^{2}\right],
\end{aligned}
$$

where $\Delta$ is the strain dilatation, $\gamma$ the shear strain, and $\alpha$ and $\kappa_{i}$ are combinations of the elastic moduli all as defined in Paper II. The second term is strictly negative and represents the weakening of the rock due to the crack porosity which is proportional to $\bar{\psi}$, the volume average of the positive field $\psi=|\varphi|$. Therefore, this mean-field Hamiltonian is maximum when $\bar{\psi}=0$, which uniquely corresponds to the uniform intact state $\psi=\varphi=0$.

\section{Interaction term}

The interesting term is the interaction energy $E^{\mathrm{int}}$. As defined in the summary of Paper II (the reader should consult this summary for the definitions of all the terms in what follows), $E^{\text {int }}$ is a sum over wave numbers $\boldsymbol{k}$ of orthogonal quadratic forms involving $R_{k}$ and $I_{k}$, which are vectors containing the $\boldsymbol{k}$-space Fourier modes of the order-parameter fields $\varphi$ and $\psi$. The sign of these forms is determined by the sign of the two eigenvalues of the symmetric matrices $P_{\boldsymbol{k}}$. For any $\boldsymbol{k}$, at least one of the eigenvalues is positive, since $[1,0] \cdot P_{k} \cdot[1,0]^{T}=L_{k}=\Delta^{2} \kappa_{1}^{2}\left(1-\alpha u_{k}^{2}\right)>0$, where $1 / 2<\alpha$ $<1$ and $u_{k}$ is a cosine. To determine the sign of the second eigenvalue, it is sufficient to take the determinant of $P_{k}$. Using $u_{k}^{2}+v_{k}^{2}=1$, it is straightforward to show that

$$
\operatorname{det}\left|P_{k}\right|=\Delta^{4} \kappa_{1}^{4}(1-\alpha)\left[c v_{k}+\omega\right]^{2} .
$$

This is strictly positive for every $\boldsymbol{k}$, except when

$$
v_{k}=\sin \left(2 \theta_{k}\right)=-\omega / c,
$$

in which case the determinant and second eigenvalue are zero. The vanishing of the determinant is thus independent of the norm of $\boldsymbol{k}$, and takes place at either of two conjugate angles $\theta_{k}^{+}=\arcsin (-\omega / c) / 2$ or $\theta_{k}^{-}=\pi / 2-\arcsin (-\omega / c) / 2$, where $\theta_{k}$ represents the angle between $\boldsymbol{k}$ and the crackorientation vector $\hat{\boldsymbol{e}}_{1}$. The directions in $\boldsymbol{k}$ space at which the determinant vanishes will be denoted by the unit vectors $\hat{\boldsymbol{k}}^{ \pm}$. Thus, the matrices $P_{\boldsymbol{k}}$ are positive definite; i.e., they have two strictly positive eigenvalues, except for those particular wave vectors lying along one of the two directions for which they become positive degenerate. The eigenvector of $P_{\boldsymbol{k}}$ associated with the zero eigenvalues is easily computed to be $\left[1,-M_{k} / L_{k}\right]^{T}$.

The positive-definite quadratic forms of $E^{\text {int }}$ are multiplied by a negative constant which implies that the maximum of $E^{\text {int }}$ occurs when $\tilde{\varphi}_{k}=\widetilde{\psi}_{k}=0$ for every nonzero $k$ with the exception of those $\boldsymbol{k}$ satisfying Eq. (3). At these degenerate angles, the Fourier modes of $\varphi$ and $\psi$ are related as

$$
\tilde{\varphi}_{k}=-\frac{L_{k}}{M_{k}} \widetilde{\psi}_{k}
$$

Now, the definition of the auxiliary field $\psi_{x}=\left|\varphi_{x}\right|$ imposes a series of constraints between $\tilde{\varphi}_{k}$ and $\widetilde{\psi}_{k}$. The simplest is obtained by noting that the space integrals of $\varphi^{2}$ and $\psi^{2}$ must be the same which is equivalent to

$$
\sum_{k}\left(\widetilde{\psi}_{k} \widetilde{\psi}_{-k}-\tilde{\varphi}_{k} \tilde{\varphi}_{-k}\right)=0
$$

For a crack-state maximizing $E^{\text {int }}$, this condition further requires that

$$
\left(\tilde{\varphi}_{0}^{2}-\widetilde{\psi}_{0}^{2}\right)+\sum_{\substack{k=k \hat{k}^{ \pm} \\ k \neq 0}}\left(1-\frac{M_{k}^{2}}{L_{k}^{2}}\right) \tilde{\varphi}_{k} \tilde{\varphi}_{-k}=0 .
$$

It will be seen momentarily that along the directions $\hat{\boldsymbol{k}}^{ \pm}$, the factors $1-M_{k \hat{\boldsymbol{k}}^{ \pm}}^{2} / L_{k \hat{\boldsymbol{k}}^{ \pm}}^{2}$ are equal, and that this quantity is an increasing function of the shear-strain parameter $\omega$ $=\left(\kappa_{3} \gamma\right) /\left(\Delta \kappa_{1}\right)$, starting at a strictly negative value when $\omega=0$ (no shear deformation yet applied), and reaching 0 at a particular value $\omega_{c}$. For every wave vector, $\tilde{\varphi}_{k} \tilde{\varphi}_{-k}=\left\|\tilde{\varphi}_{k}\right\|^{2}$ is trivially positive, and the definition of $\psi$ also requires that $\tilde{\varphi}_{0}^{2}-\widetilde{\psi}_{0}^{2} \leqslant 0$ for any crack state. From Eq. (6), we can conclude that for $\omega<\omega_{c}$, the only crack-states maximizing the interaction term $E^{\text {int }}$ must satisfy both $\widetilde{\varphi}_{0}^{2}=\widetilde{\psi}_{0}^{2}$ and, for every nonzero $k, \tilde{\varphi}_{k}=\widetilde{\psi}_{k}=0$. Such a maximum thus corresponds to a spatially uniform crack field.

At the degenerate point $\omega=\omega_{c}$, the set of maximizing crack states goes through a drastic change. Any nonzero Fourier mode of $\varphi$ and $\psi$ along the directions $\hat{\boldsymbol{k}}^{ \pm}$no longer modifies $E^{\text {int }}$ so long as $\widetilde{\varphi}_{0}^{2}=\widetilde{\psi}_{0}^{2}$; i.e., so long as the crack field has the same sign over the entire mesovolume. This degeneracy of $E^{\text {int }}$ at $\omega=\omega_{c}$ is at the origin of the localization phase transition.

The critical value $\omega_{c}$, and the corresponding wave vectors $\boldsymbol{k}$ for which nonzero Fourier modes of $\varphi$ and $\psi$ do not contribute to $E^{\text {int }}$, are determined from the two conditions

$$
\begin{aligned}
& \operatorname{det}\left(P_{k}\right)=0, \\
& L_{k}^{2}-M_{k}^{2}=0 .
\end{aligned}
$$


Using the solution of Eq. (7) given by Eq. (3) in the definitions of $L_{k}$ and $M_{k}$ given in the summary of Paper II, Eq. (8) then becomes an equation for $\omega_{c}$,

$$
\left[\omega_{c}^{2}-\left(c^{2}-1\right)\right]\left[\omega_{c}^{2}+\frac{(1-\alpha)}{\alpha} c^{2}\right]=0 .
$$

From the definitions of Paper II, we have $c>1$ while $1 / 2$ $<\alpha<1$. Thus, Eqs. (7) $-(8)$ can only be satisfied by

$$
\begin{gathered}
\omega=\omega_{c}^{ \pm}= \pm \sqrt{c^{2}-1}, \\
\sin \left(2 \theta_{k}\right)=-\left(\sqrt{c^{2}-1}\right) / c .
\end{gathered}
$$

With a radial confining pressure maintained constant, and a positive shear stress $\tau_{\text {axial }}>\tau_{\text {radial }}$, the strain components of the rock satisfy $\varepsilon_{\text {axial }}<\varepsilon_{\text {radial }}$ and $\varepsilon_{\text {axial }}<0$ so that $\omega$ $=\left(\kappa_{3} / \kappa_{1}\right)\left(\varepsilon_{\text {axial }}-\varepsilon_{\text {radial }}\right) /\left(\varepsilon_{\text {axial }}+\varepsilon_{\text {radial }}\right)$ is a positive and monotonically increasing function of the axial stress, until the rock possibly exhibits some positive volumetric strain (we will later show that this does not occur prior to localization), where this quantity diverges to $+\infty$ and increases further starting from $-\infty$. All of this establishes that Eqs. (7) and (8) have no solution until the first solution $\omega=\omega_{c}^{+}$is reached. At this particular strain value, nonzero Fourier modes of $\varphi$ and $\psi$ having any wave vector lying in one of the two directions defined by Eq. (11) can be added to a mesovolume with no change in the interaction energy.

For quartz as the rock mineral,

$$
\frac{\kappa_{1}}{\kappa_{3}} \omega_{c}^{+}=\left(\frac{\varepsilon_{\text {axial }}-\varepsilon_{\text {radial }}}{\varepsilon_{\text {axial }}+\varepsilon_{\text {radial }}}\right)_{c} \simeq 12,
$$

so that we find $\left(\varepsilon_{\text {axial }} / \varepsilon_{\text {radial }}\right)_{c} \simeq-1.2$ at the transition. Our model thus predicts the localization transition to occur after a sign reversal of $\varepsilon_{\text {radial }}$ but prior to the point where $\Delta=\varepsilon_{\text {axial }}$ $+\varepsilon_{\text {radial }}$ changes sign. These results are consistent with what is observed in usual triaxial mechanical experiments (e.g., [1-3]).

It can now be algebraically verified using the definitions of $L_{k}$ and $M_{k}$ given in Paper II, that $1-M_{k \hat{k}^{ \pm}}^{2} / L_{k \hat{k}^{ \pm}}^{2}$ does not depend on the norm $\boldsymbol{k}$ nor on which of the two directions $\hat{\boldsymbol{k}}^{ \pm}$ is selected. Further, it increases monotonically from a negative value to reach zero when $\omega=\omega_{c}^{+}$(facts used in obtaining the above results).

\section{B. Structure at the localization transition}

The goal here is to define the geometric nature of the states maximizing $E^{\text {int }}$ at the strain point $\omega_{c}$. Necessary conditions on the structure of the degenerate states were just given and these are easily made into sufficient conditions. First, the degenerate states must correspond to crack fields of constant sign. They thus satisfy everywhere $\psi=\varphi$ or $\psi=$ $-\varphi$ or, equivalently, $\widetilde{\psi}_{k}=\tilde{\varphi}_{k}$ or $\widetilde{\psi}_{k}=-\tilde{\varphi}_{k}$. Considering this together with the necessary conditions of Eqs. (4) and (7), requires that the degenerate states be one of two types: (1) $\varphi>0$ everywhere and the only possible nonzero Fourier modes of $\varphi$ have wave vector directions that satisfy $N_{k} / M_{k}$

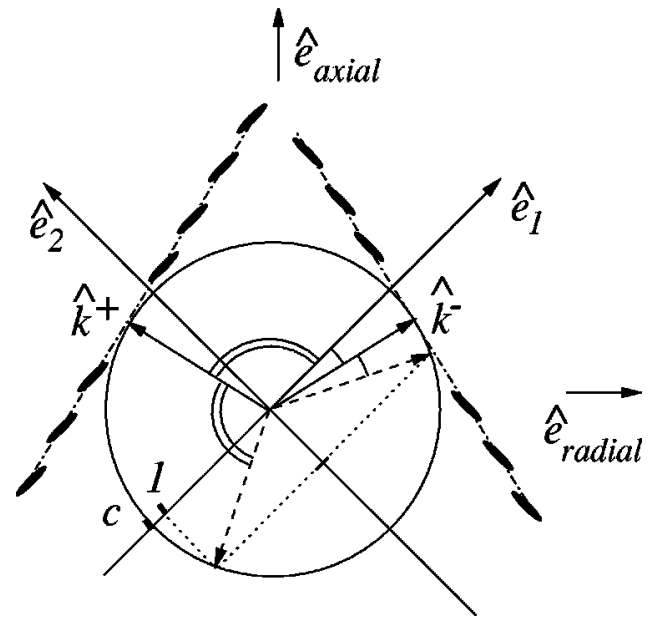

FIG. 1. A part of the conjugate bands emerging at the critical strain. The bands perpendicular to $\hat{\boldsymbol{k}}^{+}$are exclusively composed of right-inclined cracks, while those perpendicular to $\hat{\boldsymbol{k}}^{-}$contain only left-inclined cracks.

$=M_{k} / L_{k}=-1$; or (2) $\varphi<0$ everywhere and the wave vector directions satisfy $N_{k} / M_{k}=M_{k} / L_{k}=+1$. Using again the definitions of $L_{k}, M_{k}, N_{k}$ given in the summary of Paper II, the first type of degenerate mode corresponds to wave vectors satisfying $\sin \left(2 \theta_{k}\right)=-\sqrt{c^{2}-1} / c$ and $\cos \left(2 \theta_{k}\right)=-1 / c$, while the second type of mode has the same sine requirement, but an opposite value for the cosine. Using $\hat{\boldsymbol{k}}^{+}$to represent the wave vector direction corresponding to the first condition, and $\hat{\boldsymbol{k}}^{-}$the wave vector direction for the second condition, we conclude that the emergent degenerate crack states consist either of right-inclined cracks with spatial fluctuations forming bands perpendicular to $\hat{\boldsymbol{k}}^{+}$, or of leftinclined cracks forming bands perpendicular to $\hat{\boldsymbol{k}}^{-}$. Such geometry is sketched in Fig. 1.

These two sets of crack modes are conjugate to each other; i.e., symmetric to each other under inversion of the radial axis. Since they become statistically important as $\omega$ $\rightarrow \omega_{c}$, whereas the intact state or uniform states are the important states prior to $\omega_{c}$, the system spontaneously breaks its symmetry at the transition, which is characteristic of a continuous phase transition.

Further, the angle formed by these bands is at $45^{\circ}$ $-\left|\theta_{\hat{k}^{-}}\right|$from the axial direction. Using Eq. (11) and the definitions of $\kappa_{1}$ and $\kappa_{2}$ in terms of the Lamé parameters, it is found that this angle is typically between $15^{\circ}$ and $35^{\circ}$ depending on the rock mineral [4] considered which is consistent with laboratory experiments.

Finally, we note that these special crack bands that leave $E^{\text {int }}$ unchanged, make a negative contribution to the Hamiltonian through the mean-field energy $E^{\text {av }}$ that is proportional to $\bar{\psi}$. Due to the $r^{-D}$ range of elastic interactions, $E^{\text {int }}$ is independent of the norm of $\boldsymbol{k}$ (it depends only on its orientation). Thus, the spatial variation of the bands perpendicular

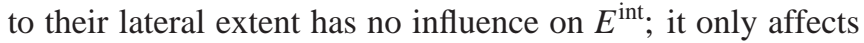
$E^{\text {av }}$ through the number of cracks present. For large systems and a narrow band of only a few cell widths, $\bar{\psi}=\Lambda \ell / \ell^{2}$ 
$=\Lambda / \ell$, where $\Lambda$ and $\ell$ are the linear size of a cell (grain) and of a mesovolume. Thus, such a thin band makes a negligible contribution to $\bar{\psi}$ for large systems, and is energetically equivalent to the intact state. However, states with numerous and/or wide bands can make a non-negligible contribution to $\bar{\psi}$ and are, therefore, less probable. So this transition indeed corresponds to "localized" structures. Only those states with a small number of small width bands along the special directions are the statistically emergent ones as is observed in actual experiments on rocks.

\section{OBTAINING THE PARTITION FUNCTION}

The sum over crack states in Eq. (1) is equivalent to the functional integration

$$
Z=\int\left(\prod_{x \in \Omega} d \varphi_{x}\right) e^{-E\left[\varphi, \boldsymbol{\varepsilon}, \boldsymbol{\varepsilon}_{m}\right] / T} .
$$

Since our Hamiltonian is expressed in terms of the Fourier modes $\tilde{\varphi}_{k}$, it is shown in standard textbooks $[5,6]$ that $Z$ further transforms to

$$
Z=\int d \tilde{\varphi}_{0} \prod_{k \in Y}\left(d \tilde{\varphi}_{k}^{R} d \tilde{\varphi}_{k}^{I}\right) e^{-E\left[\tilde{\varphi}_{k}, \boldsymbol{\varepsilon}, \boldsymbol{\varepsilon}_{m}\right] / T},
$$

where $\tilde{\varphi}_{k}^{R}$ and $\tilde{\varphi}_{k}^{I}$ are the real and imaginary part of $\tilde{\varphi}_{k}$, and $Y$ is a half space of the set of the wave vectors corresponding to the nonzero modes; i.e., corresponding in two dimensions to the discrete set $\left(k_{1}, k_{2}\right)=\left(2 \pi \ell / n_{1}, 2 \pi \ell / n_{2}\right)$ with $\left(n_{1}, n_{2}\right) \in \mathbb{Z}^{2}$. There is a small-wavelength cutoff given by $\max \left(\left|n_{1}\right| ;\left|n_{2}\right|\right)<\ell / \Lambda$ that ensures that $\varphi$ does not vary on scales smaller than that of a cell, and there is the arbitrary criterion $k_{1}>0$ made to divide this space into two symmetrical parts. Equation (13) is valid up to a multiplicative constant that has no physical importance since the properties of a system correspond to the derivatives of the free energy $F$ $=-T \ln Z$.

An analytic approximation for $Z$ is obtained by performing the functional integration over a properly chosen subset of all the possible crack states. The definition of this subset is based on what was learned in the preceeding section; namely, that among the states having a given nonzero crack occupation $\bar{\psi}$, the most probable are the uniform states, and precisely at the phase transition, certain banded states may arrive at almost no energy cost, and these emergent states also have the same sign over space. Thus, the geometrical characteristic of all such states in the "functional neighborhood" of the minimizing state is that in each one, all cracks are oriented in the same direction (either left or right). This property justifies making a so-called "constant-sign" (or "meanphase") approximation for the partition function in which only those states in which the sign does not change in space will be considered. This still includes a huge range of states in which $\varphi$ spatially varies. The excluded states in this approximation are guaranteed to have lower probabilities than the included ones and, as such, should have a negligible influence on the physical properties of the system. In this aproximation, the Fourier modes of the auxiliary $\psi$ field are trivially related to those of $\varphi$ as either $\widetilde{\psi}_{k}=\widetilde{\varphi}_{k}$ for the positive states, or $\widetilde{\psi}_{k}=-\tilde{\varphi}_{k}$ for the negative states.

We now rescale the temperature as $T=\Lambda^{D} T^{\prime} / \ell^{D}$. From the definition $T=\partial U / \partial S$ and the fact that $U$ is an energy density independent of $\ell$ while $S$ is extensive and thus increases as $\ell^{D}$, we have that $T$ scales as $\ell^{-D}$. In taking the thermodynamic limit in what follows, it is convenient to work with the purely intensive parameter $T^{\prime}$ (that is independent of $\ell$ ). Our partition function within the constant-sign approximation then takes the form

$$
\begin{aligned}
Z \simeq & \int_{+} \mathcal{D} \varphi \exp \left\{\frac{-\ell^{D}}{\Lambda^{D} T^{\prime}}\left[d+e\left(\frac{\tilde{\varphi}_{0}}{\ell^{D}}\right)+\sum_{k \in Y} w^{+}(\boldsymbol{k})\left|\frac{\tilde{\varphi}_{\mathbf{k}}}{\ell^{D}}\right|^{2}\right]\right\} \\
& +\int_{-} \mathcal{D} \varphi \exp \left\{\frac { - \ell ^ { D } } { \Lambda ^ { D } T ^ { \prime } } \left[d+e\left(\frac{\tilde{\varphi}_{0}}{\ell^{D}}\right)\right.\right. \\
& \left.\left.+\sum_{\boldsymbol{k} \in Y} w^{-}(\boldsymbol{k})\left|\frac{\tilde{\varphi}_{\mathbf{k}}}{\ell^{D}}\right|^{2}\right]\right\},
\end{aligned}
$$

where $\mathcal{D} \varphi$ is a compact notation for the functional measure $d \tilde{\varphi}_{0} \Pi_{\mathbf{k} \in \mathbf{Y}}\left(d \tilde{\varphi}_{\mathbf{k}}^{R} d \tilde{\varphi}_{\mathbf{k}}^{I}\right)$, and where $\int_{+}$and $\int_{-}$represent integration over the subsets of $\varphi$ fields that are everywhere either positive or negative. The quantities $d, e$, and $w^{ \pm}$are defined in the summary of Paper II as

$$
\begin{gathered}
d=\frac{1}{2}\left[\alpha \Delta^{2}+(1-\alpha) \gamma^{2}\right], \\
e=-\frac{\epsilon}{2}\left[\kappa_{2}\left(\Delta^{2}-q \Delta_{m}^{2}\right)+\kappa_{3}\left(\gamma^{2}-q \gamma_{m}^{2}\right)\right], \\
w^{ \pm}(\boldsymbol{k})=-\frac{\epsilon^{2}}{(1-\alpha)}\left[\left(L_{\boldsymbol{k}} \pm 2 M_{\boldsymbol{k}}+N_{\boldsymbol{k}}\right)(\boldsymbol{\varepsilon})\right. \\
\left.-q\left(L_{\boldsymbol{k}} \pm 2 M_{\boldsymbol{k}}+N_{\boldsymbol{k}}\right)\left(\boldsymbol{\varepsilon}_{m}\right)\right] .
\end{gathered}
$$

Recall that the values of the actual strain $\varepsilon$ intervening in the probability distribution and in the partition function are those along the load curve for which $\boldsymbol{\varepsilon}=\boldsymbol{\varepsilon}_{m}$. Their formal distinction only plays a role when partial derivatives of the free energy are taken to define stress. We note then that the value of $\quad w^{ \pm} \quad$ at $\quad \boldsymbol{\varepsilon}=\boldsymbol{\varepsilon}_{m} \quad$ is $\quad w^{ \pm}=-(1-q) \epsilon^{2}[1, \pm 1] \cdot P(\boldsymbol{k}) \cdot[1$, $\pm 1]^{T} /(1-\alpha)$ and since we have shown that $P$ is a positivedefinite matrix, and that the temperature $T^{\prime}$ is negative, we have that $w^{ \pm} / T^{\prime}$ in Eq. (14) is strictly positive.

The symmetry of the problem under the parity transformation (inversion of the radial axis) guarantees that both integrals in Eq. (14) are equal. Accordingly, only the first integral over positive crack states will be treated. This integral separates into products of Gaussian integrals with the only remaining coupling between the Fourier modes coming from the complicated constraints on the integration domain boundaries that are what guarantee $\varphi$ to have the same sign everywhere in real space, and $\psi$ to lie within $[0,1]$. But in order to study any singular behavior of the free energy $F$ in 
the vicinity of localization, $Z$ is determined in the thermodynamic limit in which both the system size and mesovolume size $\ell$ are taken to be infinite. In this limit, the complicated integration bounds in $\boldsymbol{k}$-space are not relevant. The integration can be carried out entirely on $\mathbb{R}^{+}$for $\tilde{\varphi}_{0} / \ell^{D}$, and $\mathbb{R}$ for each of the variables $\tilde{\varphi}_{k}^{R} / \ell^{D}, \tilde{\varphi}_{k}^{I} / \ell^{D}$ without changing the result because the contribution to these integrals in the thermodynamic limit comes from the immediate neighborhood of $\tilde{\varphi}_{0} / \ell^{D}=0$ and $\tilde{\varphi}_{k} / \ell^{D}=0$.

A technical proof of this can be obtained as follows: using $\mathrm{R}^{+}$and $\mathbb{R}$ as the integration domains produces an upper bound for $Z$ since this includes every positive crack field. A lower bound can be obtained by reducing the integration domain to a subset of the set of all positive crack fields in which $0<\tilde{\varphi}_{0}<\ell^{D}$ and $\Sigma_{k \in \Upsilon}\left(\left|\tilde{\varphi}_{k}^{R}\right|+\left|\tilde{\varphi}_{k}^{I}\right|\right) \leqslant \min \left[\tilde{\varphi}_{0} / \sqrt{2},\left(\ell^{D}\right.\right.$ $\left.\left.-\tilde{\varphi}_{0}\right) / \sqrt{2}\right]$. Integrating mode by mode over this polyhedra, the result can be shown to be asymptotically equivalent to the result of the upper bound in the limit where $\ell^{D}$ becomes infinite. This exercise is left to the attention of the reader.

Thus, no coupling between the $\boldsymbol{k}$ modes exists in the thermodynamic limit, and our approximation of the partition function takes the convenient form

$$
Z \simeq 2 z_{0} z_{1} \prod_{\boldsymbol{k} \in \Upsilon}\left[z(\boldsymbol{k})^{2}\right]
$$

where $z_{0}=e^{-\ell^{D} d / \Lambda^{D} T^{\prime}}$ and

$$
\begin{gathered}
z_{1}=\int_{x \in \mathbb{R}^{+}} d x e^{-\ell^{D} e x / \Lambda^{D} T^{\prime}}, \\
z(\boldsymbol{k})=\int_{x \in \mathbb{R}} d x e^{-\ell^{D} w^{+}(\boldsymbol{k}) x^{2} / \Lambda^{D} T^{\prime}} .
\end{gathered}
$$

In the limit $\ell \rightarrow+\infty$, these two integrals become

$$
\begin{gathered}
z_{1} \sim \Lambda^{D} T^{\prime} /\left(\ell^{D} e\right), \\
z(\boldsymbol{k}) \sim \sqrt{\pi \Lambda^{D} T^{\prime} /\left[\ell^{D} w^{+}(\boldsymbol{k})\right]} .
\end{gathered}
$$

Using Eq. (18), one then obtains the free-energy density in the thermodynamic limit

$$
F=-T^{\prime}(\ln Z) \Lambda^{D} / \ell^{D} \sim d+\frac{\Lambda^{D} T^{\prime}}{\ell^{D}} \sum_{k \in Y} \ln \left(\frac{\ell^{D} w^{+}(\boldsymbol{k})}{\Lambda^{D} T^{\prime}}\right) .
$$

The contribution $z_{1}$ has vanished in this limit due to the fact that $x \ln x \rightarrow 0$ as $x \rightarrow 0$. This is a technical consequence of the fact that for states composed of a few single bands, $\bar{\psi}$ vanishes in the thermodynamic limit, as commented upon in the previous section.

\section{SYSTEM PROPERTIES AT LOCALIZATION}

The remaining task is to link this free energy to the observables of the system by taking the partial derivatives of $F$ in the limit as localization is approached.
The two partial derivatives of primary interest are those that give the dimensionless entropy density $s=\Lambda^{D} S / \ell^{D}$ and the stress $\boldsymbol{\tau}$. From Paper I, we have

$$
-s=\left.\frac{\partial F}{\partial T^{\prime}}\right|_{\boldsymbol{\varepsilon}, \boldsymbol{\varepsilon}_{m}} \text { and } \boldsymbol{\tau}=\left.\frac{\partial F}{\partial \boldsymbol{\varepsilon}}\right|_{T^{\prime}, \boldsymbol{\varepsilon}_{m}}
$$

The free energy of Eq. (23) is rewritten by replacing the sum over the wave vectors $\Sigma_{k \in Y}$ with a continuous integral $\ell^{D} /(2 \pi)^{D} \int_{0}^{2 \pi / \Lambda} k d k \int_{0}^{\pi} d \theta$. After performing the trivial integration over $d k$ we have

$$
F=d+\frac{T^{\prime}}{2}\left[I-\pi \ln \left(-\frac{\Lambda^{D} T^{\prime}}{\ell^{D}}\right)\right]
$$

where $I$ is the integral

$$
I=\int_{-\pi / 2}^{\pi / 2} \ln \left(-w^{+}\right) d \theta
$$

The integrand $w^{+}$is a temperature-independent strain function so that $-\partial F / \partial T^{\prime}$ gives

$$
s=-\frac{I}{2}+\frac{\pi}{2}\left[1+\ln \left(-\frac{\Lambda^{D} T^{\prime}}{\ell^{D}}\right)\right]
$$

while from $F=U-T^{\prime} s$

$$
U=d+\frac{\pi}{2} T^{\prime}
$$

Since $d$ represents the linear elastic response of an intact rock, and $T^{\prime}$ decreases from zero to negative values as damage accumulates, this expression shows that the average energy decreases due to the presence of cracks and is thus consistent with the negative curvature of the strain/stress load curve observed experimentally.

Before addressing how $s$ and $F$ (and their derivatives) behave at localization, we first establish the stress and temperature behavior at localization.

\section{A. Mechanical behavior at localization}

Consider the stress components $\sigma=-2 \partial F / \partial \gamma$ and $p=$ $-2 \partial F / \partial \Delta$, where $\sigma$ (shear stress) and $p$ (pressure) are both positive and related to the axial and radial stress components as

$$
-\sigma=\tau_{a}-\tau_{r}, \quad \text { and }-p=\tau_{a}+\tau_{r}
$$

In standard laboratory experiments, the axial stress $\tau_{a}$ varies while the radial stress $\tau_{r}=-p_{r}$ is kept constant. The strain components $\gamma$ (shear strain) and $\Delta$ (dilatation) are similarly related to the axial and radial strain as

$$
\gamma=\varepsilon_{a}-\varepsilon_{r}, \quad \text { and } \Delta=\varepsilon_{a}+\varepsilon_{r} .
$$

Using the definition of $w^{+}$[Eq. (17)] along with the definitions of $L_{k}, M_{k}$, and $N_{k}$ given in the summary of Paper II, 
we differentiate the integral $I$ with respect to the actual strain variables, evaluate along the load path $\left(\Delta_{m}=\Delta\right.$ and $\gamma_{m}$ $=\gamma$ ), use the definition $\omega=\omega_{3} \gamma / \Delta$ with the new constant $\omega_{3}=\kappa_{3} / \kappa_{1}$ and make the change of integration variables $z$ $=\tan ^{-1} \theta$ to obtain exactly

$$
\begin{gathered}
\partial_{\gamma} I=\frac{\omega_{3}}{(1-q) \Delta} \partial_{\omega} I, \\
\partial_{\Delta} I=\frac{1}{(1-q) \Delta}\left(2 \pi-\omega \partial_{\omega} I\right),
\end{gathered}
$$

where $q=1-1 /(k+2)$ is the constant associated with the exponent $k \geqslant 0$ of the quenched disorder distribution, and the integral $\partial_{\omega} I$ is defined

$$
\partial_{\omega} I=\int_{-\infty}^{+\infty} \frac{\partial_{\omega} g}{g} \frac{d z}{1+z^{2}}
$$

with $g(\omega, z)$ given by

$$
\begin{aligned}
g(\omega, z)= & {\left[1-\alpha-2(1-\alpha) c+(1-\alpha) c^{2}+\omega^{2}\right] z^{4} } \\
& +[4 \alpha \omega+4(1-\alpha) c \omega] z^{3}+\left[2+2 \alpha+2(1-\alpha) c^{2}\right. \\
& \left.-2(2 \alpha-1) \omega^{2}\right] z^{2}+[-4 \alpha \omega+4(1-\alpha) c \omega] z+1 \\
& -\alpha+2(1-\alpha) c+(1-\alpha) c^{2}+\omega^{2} .
\end{aligned}
$$

Thus, the shear stress and pressure can be written as

$$
\begin{gathered}
\sigma=-2(1-\alpha) \gamma-\frac{T^{\prime}}{\Delta} \frac{\omega_{3}}{(1-q)} \partial_{\omega} I(\omega), \\
-p=2 \alpha \Delta+\frac{T^{\prime}}{(1-q) \Delta}\left[2 \pi-\omega \partial_{\omega} I(\omega)\right] .
\end{gathered}
$$

The integral $\partial_{\omega} I$ is solved using the residue theorem once the roots $z$ of the quartic $g(\omega, z)$ have been found.

This quartic decomposes into the exact form

$$
\begin{gathered}
g(\omega, z)=[z-\zeta(\omega)]\left[z-\zeta^{*}(\omega)\right] u(\omega, z), \\
u(\omega, z)=\rho(\omega)[z-\xi(\omega)]\left[z-\xi^{*}(\omega)\right],
\end{gathered}
$$

where the star indicates taking the complex conjugate. The roots $\zeta(\omega)$ and $\zeta^{*}(\omega)$ both merge to the real axis in the approach to localization $\omega \rightarrow \omega_{c}$, while the other two roots $\xi(\omega)$ and $\xi^{*}(\omega)$ remain complex at localization.

There are thus three simple poles $\zeta(\omega), \xi(\omega)$, and $i$ contributing to $\partial_{\omega} I$ if the loop is closed in the upper-half $z$ plane so that the residue theorem yields

$$
\begin{aligned}
\frac{\partial_{\omega} I}{\pi}= & \frac{\partial_{\omega} g(\zeta)}{\operatorname{Im}\{\zeta\} u(\zeta)\left[1+\zeta^{2}\right]}+\frac{\partial_{\omega} g(\xi)}{\rho \operatorname{Im}\{\xi\}[\xi-\zeta]\left[\xi-\zeta^{*}\right]\left[1+\xi^{2}\right]} \\
& +\frac{\partial_{\omega} g(i)}{[i-\zeta]\left[i-\zeta^{*}\right] u(i)},
\end{aligned}
$$

where Im designates taking the imaginary part. We are interested in evaluating this integral (and therefore, the roots $\zeta$ and $\xi$ and the function $\rho$ ) only in the approach to localization; i.e., when $\delta \omega=\omega-\omega_{c}$ can be considered small. In this limit, the second and third terms of Eq. (38) (the residues from $\xi$ and $i$ ) have numerators and denominators that are both order 0 in $\delta \omega$ so that it suffices to know the behavior

$$
\begin{aligned}
& \xi(\omega)=\xi_{0}+\xi_{1} \delta \omega, \\
& \rho(\omega)=\rho_{0}+\rho_{1} \delta \omega .
\end{aligned}
$$

However, the residue related to $\zeta$ is proportional to $\delta \omega$ in both the numerator and the denominator which requires knowledge of this root to second order

$$
\zeta(\omega)=\zeta_{0}+\zeta_{1} \delta \omega+\zeta_{2} \delta \omega^{2}
$$

The various strain-independent constants $\xi_{i}, \rho_{i}$, and $\zeta_{i}$ are all known groupings of the elastic constants derived from Eqs. (33), (36), and (37). The final result for the integral after an enormous algebraic reduction is

$$
\partial_{\omega} I=I_{c}+I_{1} \delta \omega,
$$

where the constants $I_{c}$ and $I_{1}$ are exactly

$$
I_{c}=2 \pi \frac{\sqrt{c^{2}-1}}{c^{2}} \quad \text { and } \quad I_{1}=2 \pi \frac{2-c^{2}}{c^{4}} .
$$

\section{Stress and strain at localization}

The shear stress and pressure may be written as

$$
\sigma=\sigma_{0}+\sigma^{\text {int }} \text { and } p=p_{0}+p^{\text {int }},
$$

where $\sigma_{0}=-2(1-\alpha) \gamma$ and $p_{0}=-2 \alpha \Delta$ are the trivial linear variations of the uncracked material. We have just shown that at localization $(\delta \omega=0)$, the nontrivial shear stress due to cracks and crack interaction is

$$
\sigma_{c}^{\mathrm{int}}=-\frac{2 \pi \omega_{3} T_{c}^{\prime}}{(1-q) \Delta_{c}} \frac{\sqrt{c^{2}-1}}{c^{2}}<0,
$$

while the nontrivial pressure is

$$
p_{c}^{\mathrm{int}}=-\frac{2 \pi T_{c}^{\prime}}{(1-q) \Delta_{c} c^{2}}<0 .
$$

That these critical values are both negative follows because $T_{c}^{\prime}$ (scaled temperature at localization) is negative and $\Delta_{c}$ (total dilatation at localization) will soon be shown to be negative. Equations (44) and (45) say that the presence of cracks has lowered both shear stress and pressure relative to an intact material at the same strain. This is indeed what is observed in experiments.

To quantify the nature of $\Delta_{c}$, we use that the confining pressure $p_{r}$ is a known positive constant in standard experiments on rocks so that 


$$
p_{r}=-\alpha \Delta_{c}+(1-\alpha) \gamma_{c}-\frac{T_{c}^{\prime}\left[2 \pi-\left(\omega_{c}+\omega_{3}\right) I_{c}\right]}{2(1-q) \Delta_{c}}
$$

Together with $\omega_{c}=\omega_{3} \gamma_{c} / \Delta_{c}$, this represents an equation for $\Delta_{c}$

$$
\left[\alpha-(1-\alpha) \frac{\omega_{c}}{\omega_{3}}\right] \Delta_{c}^{2}+p_{r} \Delta_{c}+\frac{T_{c}^{\prime}\left[2 \pi-\left(\omega_{c}+\omega_{3}\right) I_{c}\right]}{(1-q)}=0 .
$$

Because $T^{\prime}$ varies with strain, we have that $T_{c}^{\prime}$ is also a function of $\Delta_{c}$ so that Eq. (47) is more than a simple quadratic in $\Delta_{c}$. To obtain an order-of-magnitude estimate of $T_{c}^{\prime}$, we use the approximate temperature expression based on noninteracting cracks,

$$
\begin{aligned}
\frac{1}{T_{c}^{\prime}}= & -\frac{2 \Lambda^{2}}{d_{m}^{2}(1-q)\left[\kappa_{2}+\kappa_{3}\left(\omega_{c} / \omega_{3}\right)^{2}\right] \Delta_{c}^{2}} \\
& \times \ln \left\{\left[\frac{2 \Gamma}{(\lambda+2 \mu) d_{m} \Delta_{c}^{2}\left[\kappa_{2}+\kappa_{3}\left(\omega_{c} / \omega_{3}\right)^{2}\right]}\right]^{q /(1-q)}-1\right\} .
\end{aligned}
$$

After putting Eq. (48) into Eq. (47), $\Delta_{c}$ is numerically determined using Newton's method. The predicted $\Delta_{c}$ is negative for the range of confining pressure $p_{r}$ of interest and remains negative for all ranges of elastic moduli found in rocks. The signs of the various terms in Eq. (47) imply that the transition happens when the temperature has sufficiently departed from zero, but is still negative. Typical results from the nu- merical evaluation are $T_{c}^{\prime} \sim-10^{-2}(\lambda+2 \mu)$, which confirms the rough estimate given in Sec. V of Paper II. The typical value for $\Delta_{c}$ is a few percent; i.e., the order of magnitude experimentally observed at peak stress [7].

The conclusion is that at localization, both dilatation $\Delta_{c}$ and shear strain $\gamma_{c}=\omega_{c} \Delta_{c} / \omega_{3}$ are negative while $\left|\gamma_{c}\right|$ $\gg\left|\Delta_{c}\right|$. This demonstrates that the radial strain $\varepsilon_{r}=\Delta_{c}-\gamma_{c}$ is positive at localization, which is also consistent with experimental observations.

\section{Stress, strain, and temperature derivatives at localization}

We now address how the stress and strain components, as well as the temperature are changing with the negative of axial strain $\varepsilon=-\varepsilon_{a}=-(\Delta+\gamma) / 2$ at localization.

In the approach to localization we write $\Delta=\Delta_{c}+\delta \Delta, \gamma$ $=\gamma_{c}+\delta \gamma$, and $T^{\prime}=T_{c}^{\prime}+\delta T^{\prime}$ using the exact differential equation for temperature to define $\delta T^{\prime}$ in what follows (not the approximation). The condition that $p_{r}$ is constant requires that

$$
\begin{gathered}
\delta \Delta\left\{-\alpha+\frac{T_{c}^{\prime}\left[2 \pi-\left(2 \omega_{c}+\omega_{3}\right) I_{c}-\left(\omega_{c}+\omega_{3}\right) \omega_{c} I_{1}\right]}{2(1-q) \Delta_{c}^{2}}\right\} \\
+\delta \gamma\left\{1-\alpha+\frac{T_{c}^{\prime} \omega_{3}\left[I_{c}+\left(\omega_{c}+\omega_{3}\right) I_{1}\right]}{2(1-q) \Delta_{c}^{2}}\right\} \\
-\frac{\delta T^{\prime}}{2(1-q) \Delta_{c}}\left\{2 \pi-\left(\omega_{c}+\omega_{3}\right) I_{c}\right\}=0,
\end{gathered}
$$

which along with $-2 \delta \varepsilon=\delta \Delta+\delta \gamma$ gives

$$
\begin{gathered}
\frac{1}{2} \frac{d \Delta}{d \varepsilon}=\frac{2(1-q)(1-\alpha) \Delta_{c}^{2} / T_{c}^{\prime}+\omega_{3}\left[I_{c}+\left(\omega_{c}+\omega_{3}\right) I_{1}\right]+\Delta_{c} /\left(2 T_{c}^{\prime}\right)\left[2 \pi-\left(\omega_{c}+\omega_{3}\right) I_{c}\right] d T^{\prime} / d \varepsilon}{-2(1-q) \Delta_{c}^{2} / T_{c}^{\prime}+2 \pi-2\left(\omega_{c}+\omega_{3}\right) I_{c}-\left(\omega_{c}+\omega_{3}\right)^{2} I_{1}}, \\
\frac{1}{2} \frac{d \gamma}{d \varepsilon}=\frac{2(1-q) \alpha \Delta_{c}^{2} / T_{c}^{\prime}-2 \pi+\left(2 \omega_{c}+\omega_{3}\right) I_{c}+\left(\omega_{c}+\omega_{3}\right) \omega_{c} I_{1}-\Delta_{c} /\left(2 T_{c}^{\prime}\right)\left[2 \pi-\left(\omega_{c}+\omega_{3}\right) I_{c}\right] d T^{\prime} / d \varepsilon}{-2(1-q) \Delta_{c}^{2} / T_{c}^{\prime}+2 \pi-2\left(\omega_{c}+\omega_{3}\right) I_{c}-\left(\omega_{c}+\omega_{3}\right)^{2} I_{1}} .
\end{gathered}
$$

To obtain an exact expression for $d T^{\prime} / d \varepsilon$ (within the context of having employed the mean-phase approximation), we use the formalism of Sec. IV A of Paper I to write

$$
\left[\partial_{T^{\prime}} U+\left(\partial_{\Delta} U+\partial_{\Delta_{m}} U+\frac{p}{2}\right) \partial_{T^{\prime}} \Delta+\left(\partial_{\gamma} U+\partial_{\gamma_{m}} U+\frac{\sigma}{2}\right) \partial_{T^{\prime}} \gamma\right] \frac{d T^{\prime}}{d \varepsilon}+\left(\partial_{\Delta} U+\partial_{\Delta_{m}} U+\frac{p}{2}\right) \partial_{\varepsilon} \Delta+\left(\partial_{\gamma} U+\partial_{\gamma_{m}} U+\frac{\sigma}{2}\right) \partial_{\varepsilon} \gamma=0 .
$$

Using Eq. (27) for $U$, we have $\partial_{T^{\prime}} U=\pi / 2, \partial_{\Delta} U=\alpha \Delta=-p_{0} / 2, \partial_{\gamma} U=(1-\alpha) \gamma=-\sigma_{0} / 2, \partial_{\Delta_{m}} U=0$, and $\partial_{\gamma_{m}} U=0$ so that the temperature derivative at localization is given by

$$
\frac{1}{2} \frac{d T^{\prime}}{d \varepsilon}=\frac{\left[\left(2 \pi-\omega_{c} I_{c}\right)(1-\alpha)+\omega_{3} I_{c} \alpha\right] 2(1-q) \Delta_{c}+\omega_{3}\left(\omega_{c}+\omega_{3}\right)\left(2 \pi I_{1}+\omega_{3} I_{c}^{2}\right) T_{c}^{\prime} / \Delta_{c}}{-\left[2 \pi-\left(\omega_{c}+\omega_{3}\right) I_{c}\right]^{2}+(1-q) \pi\left[-2(1-q) \Delta_{c}^{2} / T_{c}^{\prime}+2 \pi-2\left(\omega_{c}+\omega_{3}\right) I_{c}-\left(\omega_{c}+\omega_{3}\right)^{2} I_{1}\right]} .
$$




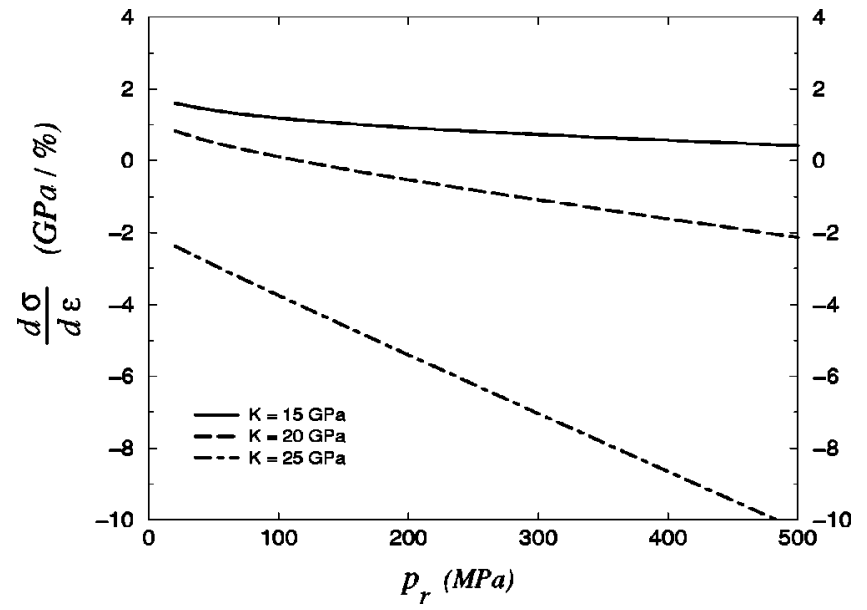

FIG. 2. The localization value of the axial tangent modulus $d \sigma / d \varepsilon$ as a function of the radial confining pressure $p_{r}$. The three curves represent different assumed bulk moduli for the mineral. The other rock properties are $\Gamma=10 \mathrm{~J} / \mathrm{m}^{2}, d_{m}=10 \mu \mathrm{m}, \mu=15 \mathrm{GPa}$, and $q=3 / 4$.

This derivative is numerically calculated to be finite and negative for the ranges of elastic moduli and radial confining pressures of interest, thus indicating that the localization transition always preceeds the phase transition where the temperature diverges to $-\infty$. Since rocks fail immediately after localization, the temperature-divergence transition is not observed in rock experiments.

Last, we determine the variation of the stress components with axial strain $\varepsilon$ at localization. Since $p_{r}$ is constant, we have that $d p / d \varepsilon=d \sigma / d \varepsilon=-d \tau_{a} / d \varepsilon$. These derivatives define the so-called "tangent modulus" given by

$$
\begin{aligned}
\frac{1}{2} \frac{d \sigma}{d \varepsilon}= & -\frac{\omega_{3}}{2(1-q)} \frac{I_{c}}{\Delta_{c}} \frac{d T^{\prime}}{d \varepsilon}+\frac{T_{c}^{\prime} \omega_{3}\left(I_{c}+\omega_{c} I_{1}\right)}{2(1-q) \Delta_{c}^{2}} \frac{d \Delta}{d \varepsilon} \\
& -\left[(1-\alpha)+\frac{T_{c}^{\prime}}{2(1-q) \Delta_{c}^{2}} \omega_{3}^{2} I_{1}\right] \frac{d \gamma}{d \varepsilon},
\end{aligned}
$$

where the derivatives $d \Delta / d \varepsilon, d \gamma / d \varepsilon$, and $d T^{\prime} / d \varepsilon$ have been given above.

In Fig. 2, we plot how $d \sigma / d \varepsilon$ varies with radial confining pressure for various values of the elastic constants. The plot shows that for a sufficiently large ratio of bulk to shear modulus, the axial pressure is always decreasing at localization, which means that it has already passed through the stress maximum. However, for sufficiently small bulk moduli and at low confining pressures, localization can also occur prior to peak stress. Thus, peak stress and localization are distinct in our theory. Localization can occur in either the hardening or softening regime depending on the bulk modulus and confining pressure. When localization occurs in the softening regime (large bulk modulus), the strain/stress curve around peak stress is necessarily an analytic (quadratic) function, whereas when it occurs in the hardening regime (small bulk modulus with small confining pressure), the peak stress presumably corresponds to a sharper variation as micro- cracks start to coalesce along a weakened band and unstable failure sets in. These predictions are consistent with the experimental observations.

\section{B. Entropy and its derivatives at localization}

The exact result $\partial_{\omega} I=I_{c}+I_{1} \delta \omega$ with $I_{c}$ and $I_{1}$ as given by Eq. (43) means that the integral $I$ of Eq. (25) is itself both finite and continuous in the limit as $\delta \omega \rightarrow 0$. Because it has further been shown that $T^{\prime}$ remains finite and continuous at localization, Eqs. (24) and (26) then show that both the free energy and the entropy (and all of their derivatives with respect to strain) remain finite and continuous as $\delta \omega \rightarrow 0$. This demonstrates exactly that the localization transition is a continuous phase transition and allows us to classify it as a critical point.

\section{CORRELATION FUNCTION}

\section{A. Derivation of a diverging correlation length}

The qualitative study of Sec. II B leads to the conclusion that the localization transition is associated with the creation of conjugate bands of coherently oriented cracks. In this final section, the statistical correlation between cracks will be quantitatively addressed.

The autocorrelation function is defined as

$$
G(\boldsymbol{x}, \boldsymbol{y})=\langle\varphi(\boldsymbol{x}) \varphi(\boldsymbol{y})\rangle-\langle\varphi(\boldsymbol{x})\rangle\langle\varphi(\boldsymbol{y})\rangle
$$

and will be determined using a standard method of statistical mechanics $[5,6,8]$. First, the Hamiltonian $E[\varphi]$ is generalized to include an additional coupling of the local field $\varphi(x)$ with an aribitrary field $J(\boldsymbol{x})$ coming from some external source

$$
E^{\prime}[\varphi, J]=E[\varphi]-\int_{x \in \Omega} d^{D} x J(x) \varphi(x)
$$

The partition function becomes then a functional of the external field

$$
Z[J]=\int \prod_{x \in \Omega}\left(d \varphi_{x}\right) e^{-E^{\prime}[\varphi, J] / T}
$$

and the averages involved in Eq. (54) are obtained by taking functional derivatives of $Z[J]$ with respect to $J$ and then letting the external field go to zero; i.e.,

$$
\begin{gathered}
\langle\varphi(\boldsymbol{x})\rangle=\lim _{J \rightarrow 0} \frac{T}{Z} \frac{\delta Z}{\delta J(\boldsymbol{x})}, \\
\langle\varphi(\boldsymbol{x}) \varphi(\boldsymbol{y})\rangle=\lim _{J \rightarrow 0} \frac{T^{2}}{Z} \frac{\delta^{2} Z}{\delta J(\boldsymbol{x}) \delta J(\boldsymbol{y})} .
\end{gathered}
$$

Since the original Hamiltonian is most easily handled in Fourier form, the external coupling will be expressed as 


$$
\begin{aligned}
& -\int_{x \in \Omega} d^{D} \boldsymbol{x} J(\boldsymbol{x}) \varphi(\boldsymbol{x}) \\
& \quad=-\frac{1}{\ell^{D}}\left(\widetilde{J}_{0} \tilde{\varphi}_{0}+2 \sum_{\boldsymbol{k} \in Y} \widetilde{J}_{\boldsymbol{k}}^{R} \widetilde{\varphi}_{\boldsymbol{k}}^{R}+2 \sum_{k \in Y} \widetilde{J}_{\boldsymbol{k}}^{I} \widetilde{\varphi}_{\boldsymbol{k}}^{I}\right),
\end{aligned}
$$

where the superscripts $R$ and $I$ refer once again to the real and imaginary parts of a complex quantity. The functional derivatives relative to $J(\boldsymbol{x})$ must then be expressed by their counterparts in Fourier space,

$$
\begin{aligned}
\frac{\delta}{\delta J(\boldsymbol{x})} & =\sum_{\boldsymbol{k} \in Y \cup\{0\}}\left(\frac{\delta \widetilde{J}_{\boldsymbol{k}}^{R}}{\delta J(\boldsymbol{x})} \frac{\partial}{\partial \widetilde{J}_{\boldsymbol{k}}^{R}}+\frac{\delta \widetilde{J}_{\boldsymbol{k}}^{I}}{\delta J(\boldsymbol{x})} \frac{\partial}{\partial \widetilde{J}_{k}^{I}}\right) \\
& =\sum_{\boldsymbol{k} \in Y \cup\{0\}}\left(\cos (\boldsymbol{k} \cdot \boldsymbol{x}) \frac{\partial}{\partial \widetilde{J}_{\boldsymbol{k}}^{R}}-\sin (\boldsymbol{k} \cdot \boldsymbol{x}) \frac{\partial}{\partial \widetilde{J}_{\boldsymbol{k}}^{I}}\right) .
\end{aligned}
$$

The modified partition function will again be determined using the constant-sign approximation, but now the presence of the external field breaks the symmetry between the sum over positive and negative crack fields, so that both terms need to be kept in the generalization of Eq. (14). This leads to a slightly more complicated version of Eq. (18) for the expression of $Z$ in the thermodynamic limit

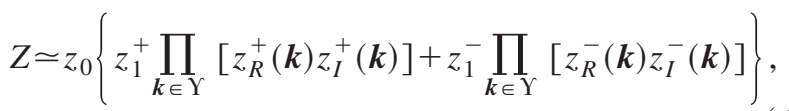

where $z_{0}$ is again the trivial intact term, and where

$$
\begin{gathered}
z_{1}^{ \pm}=\int_{x \in \mathbb{R}^{+}} d x e^{\left.-\left(e \ell^{D} \pm \widetilde{J}_{0}\right) x\right] / \Lambda^{D} T^{\prime}}, \\
z_{R}^{ \pm}(\boldsymbol{k})=\int_{x \in \mathbb{R}} d x e^{-\left[-2 \widetilde{J}_{k}^{R} x+\ell^{D} w^{ \pm}(\boldsymbol{k}) x^{2}\right] / \Lambda^{D} T^{\prime}},
\end{gathered}
$$

with $z_{I}^{ \pm}(\boldsymbol{k})$ having the same form as $z_{R}^{ \pm}(\boldsymbol{k})$ after replacing $\widetilde{J}_{\boldsymbol{k}}^{R}$ with $\widetilde{J}_{k}^{I}$. In the following, the forms implying derivatives with respect to $\widetilde{J}_{\boldsymbol{k}}^{I}$ are to be implicitly understood as having the same forms as their counterparts with respect to $\widetilde{J}_{\boldsymbol{k}}^{R}$ (these imaginary components will not be explicitly written out).

The integrals are easily performed giving

$$
\begin{gathered}
z_{1}^{ \pm}=\Lambda^{D} T^{\prime} /\left[e \ell^{D} \pm \widetilde{J}_{0}\right] \\
z_{R}^{ \pm}(\boldsymbol{k})=\exp \left[\frac{\left(\widetilde{J}_{\boldsymbol{k}}^{R}\right)^{2}}{\Lambda^{D} \ell^{D} w^{ \pm}(\boldsymbol{k}) T^{\prime}}\right] \sqrt{\frac{\pi \Lambda^{D} T^{\prime}}{\ell^{D} w^{ \pm}(\boldsymbol{k})}} .
\end{gathered}
$$

The first derivatives of $Z$ with respect to the external field are then

$$
\begin{aligned}
\frac{\partial Z}{\partial \widetilde{J}_{0}}= & z_{0}\left\{-\frac{\Lambda^{D} T^{\prime}}{\left(e \ell^{D}+\widetilde{J}_{0}\right)^{2}} z_{1}^{+} \prod_{\boldsymbol{k} \in \Upsilon}\left[z_{R}^{+}(\boldsymbol{k}) z_{I}^{+}(\boldsymbol{k})\right]\right. \\
& \left.+\frac{\Lambda^{D} T^{\prime}}{\left(e \ell^{D}-\widetilde{J}_{0}\right)^{2}} z_{1}^{-} \prod_{\boldsymbol{k} \in \Upsilon}\left[z_{R}^{-}(\boldsymbol{k}) z_{I}^{-}(\boldsymbol{k})\right]\right\}, \\
\frac{\partial Z}{\partial \widetilde{J}_{\boldsymbol{k}}^{R}}= & z_{0}\left\{z_{1}^{+} \prod_{\boldsymbol{k} \in \Upsilon} \frac{2 \widetilde{J}_{\boldsymbol{k}}^{R}}{\Lambda^{D} \ell^{D} w^{+}(\boldsymbol{k}) T^{\prime}}\left[z_{R}^{+}(\boldsymbol{k}) z_{I}^{+}(\boldsymbol{k})\right]\right. \\
& \left.+z_{1}^{+} \prod_{\boldsymbol{k} \in \Upsilon} \frac{2 \widetilde{J}_{\boldsymbol{k}}^{R}}{\Lambda^{D} \ell^{D} w^{-}(\boldsymbol{k}) T^{\prime}}\left[z_{R}^{-}(\boldsymbol{k}) z_{I}^{-}(\boldsymbol{k})\right]\right\} .
\end{aligned}
$$

Letting the external field go to zero, both of these terms disappear, so that using the chain rule of Eq. (60), the average of the crack variable $\varphi$ at any point $\boldsymbol{x}$ in a mesovolume is given by Eq. (57) to be

$$
\langle\varphi(x)\rangle=0
$$

As expected, there is no spontaneous symmetry breaking prior to the transition.

Consequently, the autocorrelation function reduces to only the second derivatives of $Z$ in Eq. (58). Differentiating Eqs. (66)-(67) with respect to $\widetilde{J}_{0}, \widetilde{J}_{\boldsymbol{k}^{\prime}}^{R}$ and $\widetilde{J}_{\boldsymbol{k}^{\prime}}^{I}$, and taking the limit where $J$ goes uniformly to zero leads to

$$
\begin{aligned}
\frac{\partial^{2} Z}{\partial \widetilde{J}_{\boldsymbol{k}}^{R} \partial \widetilde{J}_{\boldsymbol{k}^{\prime}}^{R}}=\frac{\partial^{2} Z}{\partial \widetilde{J}_{\boldsymbol{k}}^{I} \partial \widetilde{J}_{\boldsymbol{k}^{\prime}}^{I}}=\left[\frac{1}{w^{+}(\boldsymbol{k})}+\frac{1}{w^{-}(\boldsymbol{k})}\right] \frac{2 Z_{0} \delta_{\boldsymbol{k} \boldsymbol{k}^{\prime}}}{\Lambda^{D} \ell^{D} T^{\prime}}, \\
\frac{\partial^{2} Z}{\partial \widetilde{J}_{0}^{2}}=\frac{2 \Lambda^{D} T^{\prime}}{\ell^{3 D} e^{3}} Z_{0},
\end{aligned}
$$

where $Z_{0}=Z[0]$ is the original partition function without external source. All the remaining cross derivatives go to zero,

$$
\frac{\partial^{2} Z}{\partial \widetilde{J}_{\boldsymbol{k}}^{R} \partial \widetilde{\boldsymbol{J}}_{\boldsymbol{k}^{\prime}}^{I}}=\frac{\partial^{2} Z}{\partial \widetilde{J}_{\boldsymbol{k}}^{R} \partial \widetilde{J}_{0}}=\frac{\partial^{2} Z}{\partial \widetilde{J}_{\boldsymbol{k}}^{I} \partial \widetilde{J}_{0}}=0
$$

Through the chain rules of Eq. (60), these equalities show that the autocorrelation function has the form $G(x ; y)=G(x$ $-\boldsymbol{y})$ due to the symmetry of the problem under translation for an infinite system. The Fourier transform $G(\boldsymbol{x} ; \boldsymbol{y})$ $=\Sigma_{\boldsymbol{k}} \widetilde{G}_{\boldsymbol{k}} e^{i \boldsymbol{k} \cdot(\boldsymbol{x}-\boldsymbol{y})} / \ell^{D}$ is thus given by

$$
\widetilde{G}_{\boldsymbol{k}}=2 \Lambda^{D} T^{\prime}\left[\frac{1}{w^{+}(\boldsymbol{k})}+\frac{1}{w^{-}(\boldsymbol{k})}\right]
$$

when $\boldsymbol{k} \neq 0$. The special value $\widetilde{G}_{0}=2 \Lambda^{3 D} T^{\prime 3} / e^{3} \ell^{3 D}$ does not play any role in the thermodynamic limit.

In real space, the autocorrelation function is obtained by an inverse Fourier transform:

$$
G(\boldsymbol{x})=\frac{1}{4 \pi^{2}} \int_{0}^{2 \pi / \Lambda} k d k \int_{0}^{2 \pi} d \theta \widetilde{G}(\boldsymbol{k}) e^{i \boldsymbol{k} \cdot \boldsymbol{x}}
$$


Using $\widetilde{G}(-\boldsymbol{k})=\widetilde{G}(\boldsymbol{k})$ which is a consequence of $w^{ \pm}$being $\pi$-periodic functions, and working in polar coordinates $\boldsymbol{x}$ $=\left(x, \theta_{x}\right)$ and $\boldsymbol{k}=(k, \theta)$, the angular integral is divided into two symmetric domains which gives

$$
G\left(x, \theta_{x}\right)=\int_{0}^{2 \pi / \Lambda} \frac{k d k}{2 \pi^{2}} \int_{\theta_{x}}^{\theta_{x}+\pi} d \theta \widetilde{G}(k, \theta) \cos \left[k x \cos \left(\theta-\theta_{x}\right)\right] .
$$

Since $w^{ \pm}$and therefore $\widetilde{G}$ only depend on the angular part of $\boldsymbol{k}$, the integral over $k=|\boldsymbol{k}|$ yields

$$
\begin{aligned}
G\left(x, \theta_{x}\right)= & \int_{\theta=\theta_{x}}^{\theta_{x}+\pi} d \theta \frac{\widetilde{G}(\theta)}{2 \pi^{2}} \\
& \times\left\{\frac{2 \pi}{\Lambda x \cos \left(\theta-\theta_{x}\right)} \sin \left(\frac{2 \pi x \cos \left(\theta-\theta_{x}\right)}{\Lambda}\right)\right. \\
& \left.+\frac{1}{x^{2} \cos ^{2}\left(\theta-\theta_{x}\right)}\left[\cos \left(\frac{2 \pi x \cos \left(\theta-\theta_{x}\right)}{\Lambda}\right)-1\right]\right\} .
\end{aligned}
$$

For $x \gg \Lambda$, this integral is dominated by a neighborhood of $\theta=\theta_{x}+\pi / 2$, of angular size $c_{1} \Lambda / x$ with $c_{1}$ a constant of order unity. The function $\widetilde{G}(\theta)$ is almost constant over this small neighborhood, and this integral can be well approximated as

$$
G\left(x, \theta_{x}\right)=\frac{\widetilde{G}\left(\theta_{x}+\pi / 2\right)}{\Lambda^{2}} I_{G}\left(\frac{x}{\Lambda}\right),
$$

with the dimensionless integral $I_{G}$ defined as

$$
\begin{aligned}
I_{G}(u)= & 2 \pi^{2} \int_{\theta=0}^{\pi} d \theta\left\{\frac{2 \pi \sin [2 \pi u \cos (\theta)]}{u \cos (\theta)}\right. \\
& \left.+\frac{\{\cos [2 \pi u \cos (\theta)]-1\}}{u^{2} \cos ^{2}(\theta)}\right\} .
\end{aligned}
$$

An asymptotic study of this oscillating integral for $u \gg 1$ shows that $I_{G}(u) \sim c_{2} / u$, with $c_{2}$, a positive constant of order unity. Reformulating Eq. (68) with

$$
h(\theta)=2 c_{2} T^{\prime}\left[\frac{1}{w^{+}(\theta+\pi / 2)}+\frac{1}{w^{-}(\theta+\pi / 2)}\right]
$$

gives the real space autocorrelation function in the form

$$
G\left(x, \theta_{x}\right) \sim h\left(\theta_{x}\right) \frac{\Lambda}{x} .
$$

This establishes that along any direction, the autocorrelations decay as $\Lambda / x$ (for two points separated by a significant number of grains, $x \gg \Lambda$ ).

Concerning the angular dependence of $G$, the symmetry of the system under parity leads to $w^{-}(\theta)=w^{+}(\pi / 2-\theta)$ [which can also be verified directly from the definitions of $w^{ \pm}$and the dependencies of $L_{k}, M_{k}, N_{k}$ on $u_{k}=\cos (2 \theta)$ and $v_{k}=\sin (2 \theta)$ given in paper II, together with the fact that the parity symmetry keeps $v$ constant but changes the sign of $u$ ].

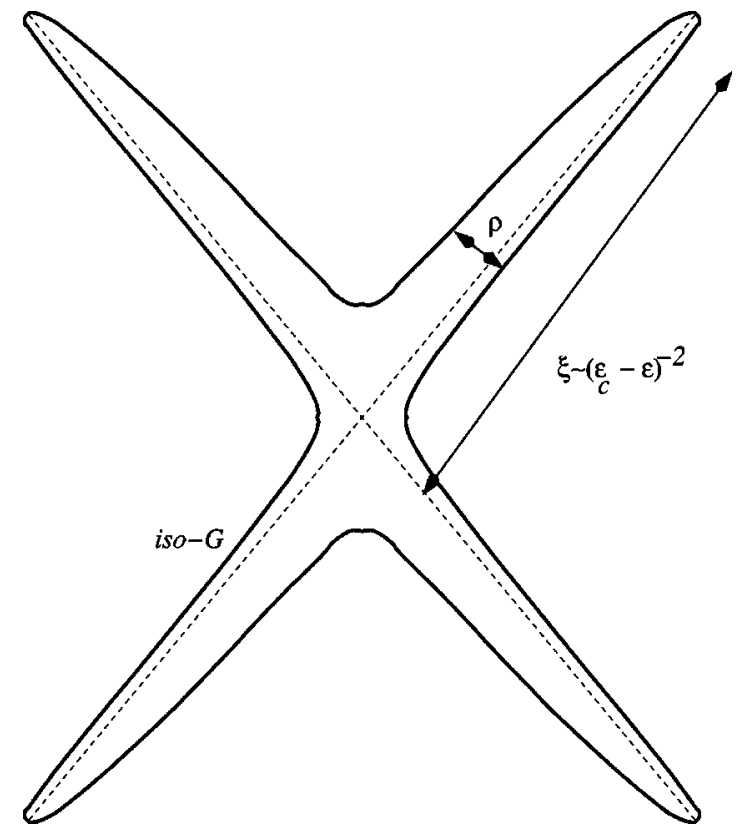

FIG. 3. Form of an isoautocorrelation curve in the approach to the localization transition.

This, along with the $\pi$ periodicity of $w^{ \pm}$, shows that $G$ is symmetric under parity; i.e., $G\left(x, \pi / 2-\theta_{x}\right)=G\left(x, \theta_{x}\right)$.

The angular dependence is best shown by considering curves of isocorrelations $G\left(x, \theta_{x}\right)=c_{3}$, where $c_{3}$ is constant along a curve. Such curves obey $x=\Lambda h\left(\theta_{x}\right) / c_{3}$. The direct study of the function $w^{+}$shows that it admits quadratic maxima along the directions $\theta^{+}[\pi]$, scaling as $\max \left(w^{+}\right)$ $=w^{+}\left(\theta^{+}[\pi]\right)=-a(\delta \omega)^{2}$ when the transition is approached, where $a$ is a positive constant. This comes from the fact that $E^{\text {int }}$ is degenerate exclusively for the critical angles $\theta^{ \pm}$, at reduced strain $\omega_{c}$. Outside a small neighborhood of $\theta^{+}[\pi]$, $w^{+}$remains bounded. The definition of $h$ and the exchange under parity of $w^{ \pm}$shows then that such an isocorrelation curve has four branches (spikes) along the directions $\pm \theta^{+}$ $\pm \pi / 2$, whose extent $\xi$ diverge to $+\infty$ as

$$
\xi \sim 2 \Lambda\left(-T^{\prime}\right) \frac{c_{2}}{a c_{3}}\left(\omega_{c}-\omega\right)^{-2} \sim c_{4} \Lambda\left(\varepsilon_{c}-\varepsilon\right)^{-2} .
$$

The fact that $w^{+}$remains bounded outside any small neighborhood of $\theta^{+}[\pi]$ also means that the width $\rho$ of the branches remains finite; i.e., that the aspect ratio of the branches $\xi / \rho$ also diverges as $\left(\varepsilon_{c}-\varepsilon\right)^{-2}$. This is qualitatively illustrated in Fig. 3. This prediction can be interpreted as corresponding to the formation of clusters of microcracks having aspect ratios $\xi / \rho$ that diverge as the cracks organize into long thin structures along which the sample will ultimately fail to form the experimentally observed shear bands.

\section{B. Experimental measurement of $\boldsymbol{\xi}$}

It would certainly be desirable to have direct experimental verification of whether the crack bands have aspect ratio that diverge as $1 /\left(\varepsilon_{c}-\varepsilon\right)^{2}$. Unfortunately, there are many practical problems that have prevented the direct measurement of the autocorrelation function of cracks in materials like rocks. We comment here on three types of measurements that either have or could be used to quantify the autocorrelation. 
First, following ideas used by Davy and Bonnet [9] in interpreting their sandbox shear experiments, one can measure the local deformation of a large sample by covering the surface with pixels and monitoring the shear strain of each pixel. The total shear strain of the system is then approximated by taking the average over the surface pixels. If the system deformation is plotted as a function of the pixel size, it is expected that when the pixels are smaller than the emergent band structures, the system deformation will decrease as a power law of increasing pixel size as was observed by Bonnet and Davy. However, at a particular pixel size there is a crossover to a constant system deformation as pixel size increases. The pixel size at the crossover point is at least an indirect measurement of the correlation length $\xi$ above which a volume-averaged description of the system holds with properties independent of the pixel size.

Second, a direct measurement of the autocorrelation between cracks can in principle be obtained via acousticemissions monitoring [10]. However, the present resolution of this method (millimeters in centimeter-scale specimens) and the difficulty in determing the mode of the individual crack events prevents having a satisfactory sampling for statistical analysis. It seems that improvements on these present limitations are possible.

Last, by analogy with the probing of spin populations by electromagnetic waves to study the ferro/paramagnetic transitions, it should be possible to send plane sound waves through a system and measure the scattering cross section as the waves scatter from the structure of the evolving microcrack population. We have not yet obtained the rigorous connection between such a measured cross section and the Fourier transform of our autocorrelation function; however, such a relation almost certainly exists. No experimental attempts to measure the correlation function of cracking systems in this manner has been attempted to our knowledge.

\section{CONCLUSION}

We now summarize the principal results that have emerged in our study. First, we have demonstrated that at a well-defined strain point $\omega=\omega_{c}$, thin bands of coherently oriented cracks can be added to the system at no energetic cost. Such localized structures break the symmetry that held when $\omega<\omega_{c}$ and correspond to a phase transition that we named the "localization transition." It was demonstrated that the free energy $F$ and entropy of the system remain continuous and finite at the localization transition which justifies calling it a critical-point phenomena. Such continuity also demonstrates that the stress/strain behavior of the rock is entirely analytic up to and including localization. The only divergence at localization is in the second derivatives of $F$ with respect to the external field $J$. The consequence is that the correlation length (aspect ratio) of the emergent-crack clusters diverges as $\left(\omega_{c}-\omega\right)^{-2}$. Presumably, if the "meanphase" approximation had not been invoked and if orderparameter contributions proportional to $\varphi^{3}$ and higher had been retained in the Hamiltonian through a renormalization scheme, then a nontrivial exponent on this scaling law might emerge.

The mechanical behavior of the system at localization exhibits many qualities observed in actual experiments on rocks. First, the stress components at localization are reduced relative to their values if the rock had remained intact. The total dilatation $\Delta_{c}$ remains negative at localization, even though the radial strain is positive. With radial confining stress kept constant, the tangent moduli $d \sigma / d \varepsilon$ are, most normally, negative at localization indicating that the load curve has already gone through a smooth quadratic peak stress prior to localization. Nonetheless, for rocks with a sufficiently low bulk modulus and at sufficiently low confining pressures, the localization can occur in the hardening regime, presumably followed by a sharp peak stress corresponding to the unstable coalescence of cracks as the sample fails along a shear band. These results are consistent with what experimentalists observe.

Using the exact differential equation that controls the temperature in the theory, it has been demonstrated that the temperature is becoming even more negative at localization that means that the temperature is always finite at localization. Unfortunately, the exact value $T_{c}$ of the temperature at localization is difficult to obtain because it is a result of integrating the differential equation from the initial conditions. Although this could be done numerically, we have instead used an approximate value of $T_{c}$ based on a noninteracting crack model.

By far the most important signature of the localization transition is the divergence of the aspect ratio of the crack clusters. As reported, no definitive experimental work has yet been performed to test this prediction and we hope that experimentalists take this as a challenge.
[1] W.F. Brace, B.W. Paulding, Jr., and C. Scholz, J. Geophys. Res. 71, 3939 (1966).

[2] R.N. Schock, H.C. Heard, and D.R. Stephens, J. Geophys. Res. 78, 5922 (1973).

[3] C.H. Scholz, J. Geophys. Res. 73, 1417 (1968).

[4] J. Bass, in Mineral Physics and Crystallography. A Handbook of Physical Constants, edited by T. J. Ahrens (American Geophysical Union, Washington, DC, 1995).

[5] M. Le Bellac, in Des Phénomènes Critiques aux Champs de Jauge, 2nd ed. (CNRS, Paris, 1990).

[6] N. Goldenfeld, Lectures on Phase Transitions and the Renor- malization Group, Frontiers in Physics (Addison-Wesley Reading, MA, 1992).

[7] M.S. Paterson, Experimental Rock Deformation—the Brittle Field (Springer-Verlag, Berlin, 1978).

[8] J.J. Binney, N. Dowrick, A. Fisher, and M. Newman, The Theory of Critical Phenomena (Clarendon Press, Oxford, 1992).

[9] P. Davy and E. Bonnet, J. Geophys. Res. (to be published).

[10] D. Lockner, in Rock Physics and Phase Relations. A Handbook of Physical Constants, edited by T.J. Ahrens (American Geophysical Union, Washington, DC, 1995), pp. 127-147. 